\title{
SARS-CoV-2 vaccination in IBD: more pros than cons
}

\author{
Ferdinando D'Amico ${ }^{1,2}$, Christian Rabaud ${ }^{3}$, Laurent Peyrin-Biroulet $\mathbb{B}^{4}$ and \\ Silvio Danese $\mathbb{1 0}^{1,2}$
}

Data on the efficacy and safety of SARS-CoV-2 vaccines are now available, but evidence for these vaccines in those who are immunocompromised (including patients with inflammatory bowel diseases) are lacking. As vaccination begins, questions on advantages and disadvantages can be partially addressed using the experience from other vaccines or immune-mediated inflammatory disorders.

Efforts to develop a vaccine to prevent the ongoing pandemic caused by severe acute respiratory syndrome coronavirus 2 (SARS-CoV-2) infection have led to multiple vaccines available and approved for use. Current evidence shows that patients with inflammatory bowel disease (IBD) do not have an increased risk of developing SARS-CoV-2 infection and coronavirus disease 2019 (COVID-19) ${ }^{1}$. However, $30 \%$ of patients with IBD are $>65$ years of age and approximately one-third of patients with IBD have comorbidities (including high-risk factors for COVID-19 such as cardiovascular diseases and diabetes) ${ }^{1}$. In addition, patients with IBD are frequently treated with immunosuppressants, biologic agents, or small molecules that expose them to increased risk of severe or opportunistic infections. It is therefore of crucial importance to define when any vaccine is recommended and the appropriate timing of vaccination in patients with IBD, especially for those on immunosuppressive treatment. The association between IBD and vaccines has been debated for decades, but an umbrella review of meta-analyses published in 2019 showed that vaccinations are not associated with an increased risk of IBD ${ }^{2}$.

Several SARS-CoV-2 vaccines are being tested ${ }^{3}$, and the first phase III clinical trials reporting efficacy and safety of vaccines are now published (Supplementary Table 1$)^{4}$. Importantly, however, patients with IBD or those treated with immunosuppressive drugs or corticosteroids were excluded from these studies. There are several pros and cons regarding SARS-CoV-2 vaccination for patients with IBD (BOX 1), and it is unknown whether SARS-CoV-2 vaccine efficacy and safety in populations with IBD are comparable with those found in the general population, whether treatment with immunosuppressive drugs affects response to vaccination or disease activity, and what is the optimal timing for any vaccination ${ }^{5}$. Long-term outcomes of any SARS-CoV-2 vaccine are also lacking, and this question of safety is crucial as vaccines will be administered to millions of people with IBD worldwide.
To date, studies on SARS-CoV-2 vaccination in IBD populations are lacking but, in this health-emergency setting, some data on safety and efficacy could be extrapolated from previous evidence with other vaccines. A prospective study by Fiorino et al. ${ }^{6}$ investigated the efficacy of pneumococcal vaccination in 96 patients with IBD undergoing immunosuppressive therapy. Treatment with anti-TNF agents or combination therapy (anti-TNF agents plus thiopurines) was associated with an impaired immune response compared with patients treated with mesalazine (57.6\% and $62.5 \%$ versus $88.6 \%$; $P<0.05$ for both comparisons), whereas azathioprine alone did not influence the antibody titres, suggesting that vaccination should be performed before starting antiTNF therapy ${ }^{6}$. Similarly, a controlled paediatric study evaluated efficacy and safety of the influenza vaccine in 51 patients with IBD (mean age 13.9 years) and 29 healthy individuals as controls (mean age 12.7 years), measuring haemagglutinin inhibition titres before and after immunization. Those patients with IBD treated with anti-TNF drugs had a reduced response to vaccination compared with patients treated with thiopurines, steroids, or anti-inflammatory compounds (mesalazine, antibiotics, and nutritional therapy) ${ }^{7}$.

Additional data can be extrapolated from other immunemediated diseases. A randomized placebo-controlled phase II trial enrolling 383 patients with rheumatoid arthritis showed that individuals starting tofacitinib 2-3 weeks after live zoster vaccination had an antibody titre concentration comparable to that of the placebo group, in the absence of an increased risk of serious adverse events ${ }^{8}$. In rheumatoid arthritis, the response to pneumococcal and influenza vaccines was reduced in patients with ongoing tofacitinib therapy, which supports that vaccinations should be performed before starting treatment ${ }^{8}$. With regard to the association between vaccines and disease activity, the International Psoriasis Council states that there is no evidence that vaccines affect psoriasis onset or severity, supporting 
Box 1 | Considerations for SARS-CoV-2 vaccination in patients with IBD

Pros

- Protection against SARS-CoV-2 infection

- Promising safety profile

- Social responsibility (to protect those who might be vulnerable)

- Potential protection against other viruses

- Herd immunity

\section{Cons}

- Unknown long-term safety

- Unknown vaccination outcomes in IBD

- Unknown effect on IBD disease activity

- Uknown vaccination outcomes during immunosuppression

Research gaps associated with SARS-CoV-2 vaccination in IBD

- Is SARS-CoV-2 vaccine equally effective in patients with IBD and in the general population?

- Does SARS-CoV-2 vaccine affect IBD disease activity?

- Do IBD medications affect the response to SARS-CoV-2 vaccination?

- Should the antibody titre after SARS-CoV-2 vaccination be monitored? If so, what is the optimal timing for monitoring?

- Should asymptomatic and/or paucisymptomatic SARS-CoV-2-infected patients with IBD and those with COVID-19 be vaccinated? If so, at what timing after infection?

IBD, inflammatory bowel disease; SARS-CoV-2, severe acute respiratory syndrome coronavirus 2. of COVID-19 far outweighs the risk of data uncertainty in an underestimated population. In line with previous vaccine experiences, it is reasonable to assume that any vaccination should be performed prior to initiating immunosuppressive therapy ${ }^{5}$. In patients with IBD treated with immunosuppressive treatment, vaccine administration should be recommended based on a favourable risk:benefit ratio and supported by an apparently reassuring safety profile and a clinically significant risk of hospitalization, complications and death associated with SARS-CoV-2 infection ${ }^{1}$. Vaccine advantages are constituted not only by the individual and herd protection against SARS-CoV-2, but also by the potential immunity against other coronaviruses ${ }^{10}$. The management of patients with IBD who have already experienced SARS-CoV-2 infection remains to be defined and efficacy and safety of vaccination in this specific setting must be investigated. Studies are also needed to define whether the antibody titre should be monitored after vaccination and, if so, how often and for how long such monitoring should be performed.

A year has passed since SARS-CoV-2 was initially identified, but major steps have been made in research to address this dangerous threat - SARS-CoV-2 vaccine development represents a fundamental tool to control the viral spread. For this purpose, the SARS-CoV-2 vaccination programme should include the millions of people with immune-mediated diseases to protect this vulnerable population and achieve the ultimate goal of the highest possible vaccination coverage. For adequate management of patients with IBD, it is essential that gastroenterologists are appropriately updated on efficacy and safety of the SARS-CoV-2 vaccine to provide clear information and guidance to patients with IBD, improving their attitude towards vaccination and reducing the skepticism and hesitation of some individuals. Moreover, social responsibility towards vaccination should be taken into account in the decision to receive a SARS-CoV-2 vaccine. During the early stages of vaccine distribution there will be a possible disproportion between number of available vaccine doses and patients to be treated, requiring patient selection. In this scenario, patients $>65$ years and those with comorbidities (such as obesity, diabetes, and cardiovascular disease) should have priority as they are at greater risk of negative outcomes (for example, hospitalization or need for oxygen therapy), as should workers at high risk of contagion (such as health-care or frontline workers and teachers). Data collection on patients with IBD receiving SARS-CoV-2 vaccines is mandatory to ensure the best preventive strategy.

reservation to vaccinate in the IBD population and in vulnerable individuals (such as children and pregnant women) is not associated with a known contraindication (such as live virus vaccine), but as a cautionary perspective relative to uncertainty of vaccine efficacy and safety in a subgroup of individuals who were excluded from the randomized clinical trials. The British Society of Gastroenterology has recently published key recommendations strongly supporting SARS-CoV-2 vaccination in patients with IBD, underlining that the main concern in patients treated with biologic agents or small molecules is the theoretical risk of suboptimal vaccine responses rather than vaccine adverse effects. However, the risk of morbidity and mortality associated with complications
1. D’Amico, F., Danese, S. \& Peyrin-Biroulet, L. Systematic Review on Inflammatory Bowel Disease Patients With Coronavirus Disease 2019: It Is Time to Take Stock. Clin. Gastroenterol. Hepatol. Off. Clin. Pract. J. Am. Gastroenterol. Assoc. 18, 2689-2700 (2020).

2. Piovani, D. et al. Environmental Risk Factors for Inflammatory Bowe Diseases: An Umbrella Review of Meta-analyses. Gastroenterology 157, 647-659.e4 (2019).

3. Melmed, G. Y., Rubin, D. T. \& McGovern, D. P. B. Winter is coming! Clinical, immunological, and practical considerations for vaccinating patients with IBD during the COVID pandemic. Gastroenterology https://doi.org/10.1053/j.gastro.2020.10.013 (2020).

4. Polack, F. P et al Safety and Efficacy of the BNT162b2 mRNA Covid-19 Vaccine. N. Engl. J. Med. 383, 2603-2615 (2020).

5. Rahier, J. F. et al. Second European evidence-based consensus on the prevention, diagnosis and management of opportunistic infections in inflammatory bowel disease. J. Crohns Colitis $\mathbf{8}$, 443-468 (2014). 
6. Fiorino, G. et al. Effects of immunosuppression on immune response to pneumococcal vaccine in inflammatory bowel disease: a prospective study. Inflamm. Bowel Dis. 18, 1042-1047 (2012)

7. Mamula, P. et al. Immune response to influenza vaccine in pediatric patients with inflammatory bowel disease. Clin. Gastroenterol. Hepatol. Off. Clin. Pract. J. Am. Gastroenterol. Assoc. 5, 851-856 (2007).

8. Winthrop, K. L. et al. The effect of tofacitinib on pneumococcal and influenza vaccine responses in rheumatoid arthritis. Ann. Rheum. Dis. 75, 687-695 (2016).

9. Rahier, J.-F. et al. H1N1 vaccines in a large observational cohort of patients with inflammatory bowel disease treated with immunomodulators and biological therapy. Gut 60, 456-462 (2011).

10. Loos, C. et al. Evolution of Early SARS-CoV-2 and Cross-Coronavirus Immunity. mSphere 5, e00622-20 (2020).

\section{Competing interests}

L.P.-B. has served as a speaker, consultant and advisory board member fo Merck, Abbvie, Janssen, Genentech, Mitsubishi, Ferring, Norgine, Tillots, Vifor, Hospira/Pfizer, Celltrion, Takeda, Biogaran, Boerhinger-Ingelheim Lilly, H.A.C. Pharma, Index Pharmaceuticals, Amgen, Sandoz, Forward Pharma, Celgene, Biogen, Lycera, Samsung Bioepis and Theravance. S.D. has served as a speaker, consultant, and advisory board member for
Schering-Plough, AbbVie, Actelion, Alphawasserman, AstraZeneca, Cellerix, Cosmo Pharmaceuticals, Ferring Genentech Grunenthal, Johnson and Johnson, Millenium Takeda, MSD, Nikkiso Europe GmbH, Novo Nordisk, Nycomed, Pfizer, Pharmacosmos, UCB Pharma and Vifor. The remaining authors declare no competing interests.

\section{Peer review information}

Nature Reviews Gastroenterology \& Hepatology thanks G. Kaplan and the other, anonymous, reviewer(s) for their contribution to the peer review of this work.

\section{Supplementary information}

The online version contains supplementary material available at https:// doi.org/10.1038/s41575-021-00420-w.

\section{RELATED LINKS}

American College of Rheumatology: https://www.rheumatology.org/ British Society of Gastroenterology: https://www.bsg.org.uk/covid-19advice/british-society-of-gastroenterology-inflammatory-bowel-diseasesection-and-ibd-clinical-research-group-position-statement-on-sars-cov2vaccination

International Psoriasis Council: https://www.psoriasiscouncil.org/blog/ IPC-Statement-on-SARS-CoV-2-Vaccines-and-Psoriasis.htm 